\title{
THE EXISTENCE OF COMPLETE CYCLES IN REPEATED LINE-GRAPHS ${ }^{1}$
}

\author{
BY GARY CHARTRAND
}

\section{Communicated by V. Klee, March 24, 1965}

With every nonempty ordinary graph $G$ there is associated a graph $L(G)$, called the line-graph of $G$, whose points are in one-to-one correspondence with the lines of $G$ and such that two points are adjacent in $L(G)$ if and only if the corresponding lines of $G$ are adjacent. By $L^{2}(G)$, we shall mean $L(L(G))$; and, in general, $L^{k}(G)$ will denote $L\left(L^{k-1}(G)\right)$ for $k \geqq 1$, where $L^{1}(G)$ and $L^{0}(G)$ stand for $L(G)$ and $G$, respectively. The graphs $L(G), L^{2}(G), L^{3}(G)$, etc. are referred to as the repeated line-graphs of $G$. A complete cycle (or hamiltonian cycle) in a (connected) graph $G$ is a cycle containing all the points of $G$. The purpose of this note is to outline a proof of the following result, a complete proof of which will be published elsewhere.

THEOREM 1. If $G$ is a nontrivial connected graph of order $p$ (having $p$ points), and if $G$ is not a path, then $L^{n}(G)$ contains a complete cycle for all $n \geqq p-3$. Furthermore, the number $p-3$ cannot, in general, be improved.

A graph $G$ having $q$ lines, where $q \geqq 3$, is called sequential if the lines of $G$ can be ordered as $x_{0}, x_{1}, x_{2}, \cdots, x_{q-1}, x_{q}=x_{0}$ so that $x_{i}$ and $x_{i+1}, i=0,1, \cdots, q-1$, are adjacent. The next theorem follows immediately.

TheOREM 2. A necessary and sufficient condition that the line-graph $L(G)$ of a graph $G$ contain a complete cycle is that $G$ be a sequential graph.

If a graph $G$ contains a complete cycle $C$, then the lines of $C$ can be arranged in a cyclic fashion. By an appropriate "interspersing" of the lines not on $C$ (if any) among the lines which are on $C$, we can produce an ordering of all the lines of $G$ as needed to show that $G$ is sequential. This fact coupled with Theorem 2 gives the next result.

ThEOREM 3. If a graph $G$ contains a complete cycle, then $L(G)$ also contains a complete cycle.

Corollary. If a graph $G$ contains a complete cycle, then $L^{n}(G)$ contains a complete cycle for all $n \geqq 1$.

1 This research is part of a doctoral thesis written under the direction of Professor E. A. Nordhaus of Michigan State University. 
The following two lemmas can be quickly established.

LEMMA 1. If a graph $G$ has a cycle $C$ with the property that every line of $G$ is incident with at least one point of $C$, then $L(G)$ contains a complete cycle.

Lemma 2. Let $G$ be a graph consisting of a cycle $C$ and its diagonals (a diagonal of $C$ being a line which is not on $C$ but which is incident with two points of $C$ ) and $m$ paths $P_{1}, P_{2}, \cdots, P_{m}$, where (i) each path has precisely one endpoint in common with $C$ and (ii) for $i \neq j$, $P_{i}$ and $P_{j}$ are disjoint except possibly having an endpoint in common if this point is also common to $C$. Then, if the maximum of the lengths of the $P_{i}$ is $M, L^{n}(G)$ contains a complete cycle for all $n \geqq M$.

The proof of Theorem 1 is by induction on $p$ with the graphs having order 3,4 , or 5 treated individually. It is assumed then that for all connected graphs $G^{\prime}$ which are not paths and which have order $s$, where $s<p$ and $p \geqq 6, L^{n}\left(G^{\prime}\right)$ contains a complete cycle for each $n \geqq s-3$. The proof involves showing that if $G$ is a graph which is not a path and which has order $p$, then $L^{p-4}(G)$ is a sequential graph so that $L^{p-3}(G)$ contains a complete cycle (by Theorem 2) and $L^{n}(G)$ contains a complete cycle for all $n \geqq p-3$ (by the corollary to Theorem 3).

If $G$ is a cycle, the result follows directly, so without losing generality, we assume that $G$ contains a point $v$ having degree 3 or more. Let $H$ denote the connected star subgraph whose lines are all those incident with $v$, and let $Q$ denote the subgraph whose point set consists of all the points of $G$ different from $v$ and whose lines are all those which are in $G$ but not in $H$. $H$ and $Q$ have $\operatorname{deg} v$ points in common but are line disjoint. We denote the components of $Q$ by $G_{1}, G_{2}$, $\cdots, G_{k}$.

$L(H)$ is a complete subgraph of $L(G)$ and so has a cycle containing all the points of $L(H)$. If $J_{1}$ denotes $L(H)$ plus all those lines in $L(G)$ incident with one point of $L(H)$, then, by Lemma $1, H_{1}=L\left(J_{1}\right)$ has a cycle containing all the points of $H_{1}$. We let $J_{2}$ denote $L\left(H_{1}\right)$ plus any lines of $L^{2}(G)$ incident with a point of $L\left(H_{1}\right)$ and let $H_{2}=L\left(J_{2}\right)$. Once again, by Lemma $1, H_{2}$ has a cycle containing all the points of $H_{2} . J_{i}$ and $H_{i}, i=3,4, \cdots$, are defined analogously, and each $H_{i}$ has a cycle containing all the points of $H_{i}$.

Two cases are considered: (1) All the $G_{i}$ are paths or isolated points, and (2) there is at least one $G_{i}$ different from a path or an isolated point. In the first case, it follows, with the aid of Lemma 2, that $L^{p-4}(G)$ contains a complete cycle so that $L^{p-3}(G)$ contains such a cycle also. 
In the second case, we assume that the first $t$ components, $1 \leqq t \leqq k$, of $G_{1}, G_{2}, \cdots, G_{k}$ are not paths or isolated points. Clearly, each of the components $G_{1}, G_{2}, \cdots, G_{t}$ has at least 3 points. If $t<k$, the paths (or isolated points) $G_{t+1}, \cdots, G_{k}$ have orders at most $p-4$, and it is easily seen that for these components, $L^{p-4}\left(G_{i}\right)$ does not exist. $L^{p-4}(G)$ can thus be expressed as the pairwise line disjoint sum of the graphs $J_{p-4}, L^{p-4}\left(G_{1}\right), L^{p-4}\left(G_{2}\right), \cdots, L^{p-4}\left(G_{t}\right)$, where each of the graphs $L^{p-4}\left(G_{i}\right), i=1,2, \cdots, t$, has a cycle containing all the points of $L^{p-4}\left(G_{i}\right)$ by the inductive hypothesis.

Since $p \geqq 6$, it can be shown that for each $i=1,2, \cdots, t$, there is a point $u_{i}$ in $H_{p-4}$ adjacent to both endpoints of a line in $L^{p-4}\left(G_{i}\right)$. Using this result, we produce a suitable ordering of the lines of $L^{p-4}(G)$ thereby showing it to be a sequential graph.

Theorem 1 permits us to make the following definition.

Definition. Let $G$ be a nontrivial connected graph which is different from a path. The hamiltonian index of $G$, denoted by $h(G)$, is the smallest nonnegative integer $n$ such that $L^{n}(G)$ contains a complete cycle.

It now follows immediately that a graph contains a hamiltonian cycle if and only if its hamiltonian index is zero. Theorem 1 may now be restated in the following way. If $G$ is a nontrivial connected graph of order $p$ which is not a path, then $h(G)$ exists and $h(G) \leqq p-3$. To show that the bound given in Theorem 1 cannot be improved, we note that for every $p \geqq 3$, there are graphs whose hamiltonian indices are $p-3$. The graphs $G_{1}$ and $G_{2}$ shown in Figure 1 have hamiltonian indices equal to $p-3$.
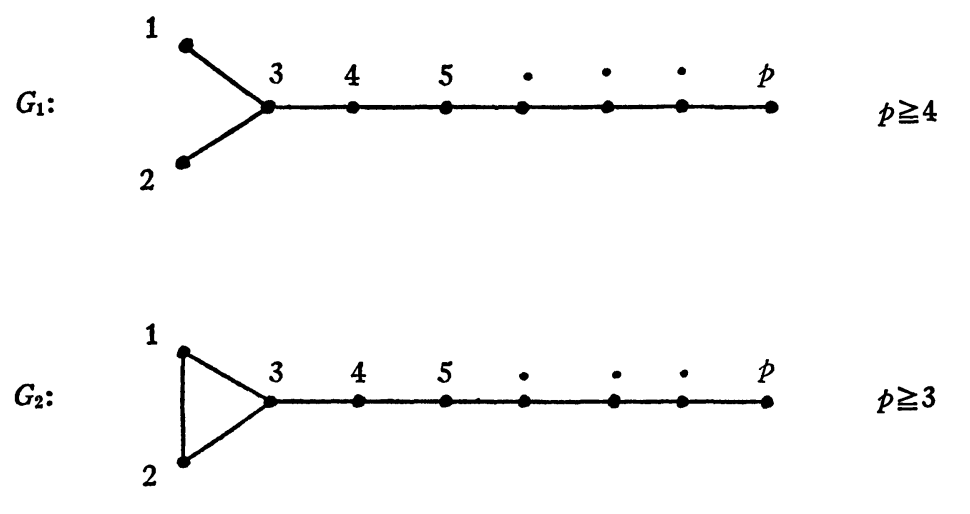

FIGURE 1

Western Michigan University 